\title{
The Emerging Role of Vascular Smooth Muscle Cell Apoptosis in Atherosclerosis and Plaque Stability
}

\author{
Murray Clarke Martin Bennett \\ Division of Cardiovascular Medicine, University of Cambridge, Addenbrooke's Hospital, Cambridge, UK
}

\section{Key Words}

Vascular smooth muscle cell · Apoptosis - Atherosclerosis • Inflammation

\begin{abstract}
Accelerated atherosclerosis is both a cause and a consequence of chronic renal failure. Vascular smooth muscle cells (VSMCs) are an important component of atherosclerotic plaques, responsible for promoting plaque stability in advanced lesions. In contrast, VSMC apoptosis has been implicated in a number of deleterious consequences of atherosclerosis, including plaque rupture, vessel remodelling, coagulation, inflammation and calcification. Although VSMC apoptosis occurs in association with these processes, its precise contribution to these diseases is unknown, given that apoptosis frequently accompanies vessel injury or alterations to flow. Using transgenic mice with selective induction of VSMC apoptosis, a recent study has precisely determined the direct consequences of VSMC apoptosis in both normal vessels and atherosclerotic plaques. Surprisingly, normal arteries can withstand huge cell losses with little change in active or passive properties. Normal vessels demonstrate highly efficient clearance of apoptotic bodies, even in the absence of professional phagocytes. In contrast, VSMC apoptosis alone is sufficient to induce multiple features of vulnerability to rupture in plaques. This study identifies VSMC apoptosis as a critical process determining plaque stability and thus the most important consequence of atherosclerosis, plaque rupture.

Copyright $\odot 2006$ S. Karger AG, Basel
\end{abstract}

\section{Introduction}

Accelerated atherosclerosis is both a common cause and consequence of chronic renal failure, and graft vasculopathy is the commonest cause of renal transplant failure. Although the amount of atherosclerosis determines the extent of tissue ischaemia, it is the quality, not the quantity of atherosclerosis that determines the most important sequelae, that of plaque rupture with subsequent organ failure. In the heart, this produces myocardial infarction, in the brain, stroke.

Atherosclerotic plaques consist of an accumulation of vascular smooth muscle cells (VSMCs), inflammatory cells (macrophages, $\mathrm{T}$ lymphocytes, dendritic cells and mast cells) underlying a dysfunctional endothelium, together with extracellular lipid, collagen and matrix. Recruitment of circulating inflammatory cells is an early event in atherosclerosis, which triggers reactive proliferation and migration of VSMCs of the vessel wall. There is also increasing evidence that circulating cells can transdifferentiate into VSMCs that comprise native atherosclerotic plaques and a variety of other vascular pathologies $[1,2]$. Most atherosclerosis is clinically silent, and consequences of atherosclerosis rarely occur before the development of advanced lesions. Many advanced plaques comprise a VSMC-rich fibrous cap overlying a lipid- and macrophage-rich necrotic core, and it is the relative proportion of these components that determines the clinical manifestations of the plaque. For example, unstable plaques (that are prone to rupture) contain a higher pro-

\section{KARGER}

Fax +4161306 1234 E-Mail karger@karger.ch www.karger.com
Prof. Martin Bennett

British Heart Foundation Professor of Cardiovascular Sciences

Division of Cardiovascular Medicine, University of Cambridge

Box 110, Addenbrooke's Hospital, Cambridge CB2 2QQ (UK)

Tel. +44 1223331 504, Fax +44 1223331 505, E-Mail mrb@mole.bio.cam.ac.uk 
portion of inflammatory cells and lipid, and a lower proportion of VSMCs than stable lesions [3]. In particular, the fibrous cap of advanced plaques is thinned from loss of VSMCs, and these thin-cap fibroatheromata are the commonest lesions that rupture to produce fatal heart attacks [4].

Until relatively recently VSMCs have been viewed as directly responsible for generating the atherosclerotic plaque, via proliferation, migration from the media and synthesis of matrix proteins [5]. Consequently huge efforts have been made to inhibit the accumulation of these cells, which in the case of stent stenosis have largely been successful. In contrast, recent reviews of atherosclerosis have emphasised the beneficial protective role of VSMCs in atherosclerosis [6]. This is based on findings that plaques in humans that have undergone plaque rupture, and directly led to heart attacks, show a paucity of VSMCs compared with stable lesions [3]. Indeed VSMCs are the only cells capable of synthesising components of the fibrous cap in plaques (the structure that separates the blood from the thrombogenic plaque interior), and whose rupture or erosion may trigger myocardial infarction.

In recent years apoptosis of VSMCs has been recognised in atherosclerosis. In early lesions apoptotic frequencies are minimal but peak in advanced plaques with both VSMCs and macrophages showing features of apoptosis [7]. This observation raised the possibility that VSMC apoptosis could promote plaque rupture by thinning the fibrous cap. Indeed, plaques from patients with unstable symptoms show higher levels of apoptosis than those with stable lesions [8]. VSMC apoptosis has also been associated with numerous other features within plaques including inflammation, calcification, thrombosis [reviewed in 9] and both negative remodelling (vessel shrinkage) $[10,11]$ and aneurysm formation [12]. VSMC apoptosis causes release of IL- $1 \alpha$ and up-regulation of monocyte-chemoattractant protein 1 (MCP-1) [13] and interleukin (IL) 8, causing infiltration of macrophages in vivo [14]. In vitro, VSMC apoptosis can promote both thrombin generation [15] and vascular calcification [16], and apoptotic vascular cells are thrombogenic both locally [17] and systemically [18] in patients, in part by the generation of microparticles.

Although these association studies are interesting, it has been very difficult to study the direct consequences of VSMC apoptosis in vivo because we lacked a model in which VSMC-specific apoptosis could be induced within the vessel wall. In addition, given that apoptotic frequencies in advanced plaques are low $(<1 \%)$, it is not clear how relevant experiments that induce high levels of VSMC apoptosis in vitro, and in the absence of other cell types, are to the real vessel wall. To address this problem, we have generated transgenic mice whose VSMCs undergo inducible apoptosis in both the media and intima. Apoptotic frequencies were increased acutely, but only low levels of apoptosis could be found following chronic administration. Despite long-term diphtheria-toxin (DT)induced apoptosis resulting in a 50-70\% VSMC loss, normal arteries showed no inflammation, reactive proliferation, thrombosis, remodelling or aneurysm formation. In contrast, VSMC apoptosis in atherosclerotic plaques of $S M 22 \alpha-h D T R / A p o E-/-$ mice induced marked fibrous cap thinning, loss of collagen and matrix, accumulation of cell debris and intense intimal inflammation. We concluded that VSMC apoptosis is 'silent' in normal arteries, which demonstrate a significant capacity to withstand cell loss. In contrast, VSMC apoptosis alone is sufficient to induce features of plaque vulnerability in atherosclerosis [19].

So what does this study mean for the role of VSMC apoptosis in both the cause and consequence of atherosclerosis, and in particular, plaque rupture? Firstly, arteries appear to be able to withstand a large loss of medial VSMCs without any change in structure or their active or passive properties. This implies that normal vessels possess a great reserve of contractile capacity, enabling vessels to generate identical maximum tensions with only $30-50 \%$ of their normal cell component. In addition, contractile tensions generated at each given concentration of all agonists tested were also identical, suggesting a highly dynamic and regulated adaptive response to cell loss. The mechanisms behind this adaptation resulting in normal active contractile properties are currently unclear. Importantly, in the absence of other stimuli, VSMC apoptosis alone was unable to induce local thrombosis or calcification in the vessel wall, and did not promote remodelling or aneurysm formation. The resistance of normal vessels to deleterious effects of manipulating VSMC apoptosis is important, as it implies that therapies aimed at VSMC apoptosis will be relatively disease specific.

Secondly, whilst high levels of VSMC apoptosis could be induced within days in normal arteries in these mice, apoptotic bodies are rapidly cleared by surrounding VSMCs without any apparent infiltration by leucocytes or inflammation. Thus VSMCs are demonstrated to have significant phagocytic capacity, and clearance does not require recruitment of professional phagocytes. Clearance is so efficient that all cell remnants from apoptosis of approximately $20 \%$ of the medial VSMCs is cleared within a week of stopping treatment, and without any 
obvious release of pro-inflammatory cellular contents or secondary necrosis. The lack of macrophages may not be surprising given that an infiltrating macrophage would have to degrade and migrate through multiple layers of the surrounding internal elastic lamina to be able to physically reach and phagocytose an apoptotic VSMC. However, the efficient clearance of VSMCs by VSMCs was not predicted. Again, although the ability of VSMCs to engulf apoptotic VSMCs in vitro has been shown to require phosphatidylserine exposure [20], the mechanism and receptors by which VSMCs so efficiently clear the bodies are unclear.

The most remarkable results came from studies inducing VSMC apoptosis in atherosclerotic plaques (fig. 1). VSMC apoptosis within atherosclerotic plaques induced multiple features of plaque vulnerability (to rupture), including fibrous cap thinning, increased necrotic cores, loss of structural proteins, and foci of intense inflammation. Increased levels of apoptotic debris were commonly observed suggesting a defect in clearance within the plaque micro-environment, as has been suggested in humans [21]. This defect, leading to secondary necrosis of the apoptotic bodies, may be the cause of the localised MCP-1 and increased systemic IL- 6 that we find within the DT-treated transgenic animals, which in turn may promote monocyte recruitment into the lesions. Interestingly, despite a large accumulation of macrophages localised around apoptotic VSMC debris within the foci, the former seem unable to clear the cell remnants, further suggesting a widespread 'mechanistic' defect in clearance. In the acute setting, VSMC apoptosis was unable alone to promote luminal thrombosis, even when surrounded by macrophages that generate tissue factor [22], although local thrombosis was seen. In contrast, calcification was not seen after acute apoptosis. It is possible that these effects require chronic apoptosis in plaques, and therefore were not seen in acute lesions, but this will only be clarified by further studies.

\section{Direct Consequences of VSMC Apoptosis}

This study clarifies what is and what is not a direct consequence of VSMC apoptosis both in the normal vessel wall and the advanced atherosclerotic plaque over the time frame of the study. For example, there has been considerable debate as to the biological significance of the low levels of apoptosis seen in vessels, even in atherosclerotic plaques. In a process that takes decades to develop, low frequencies of both cell proliferation and cell death

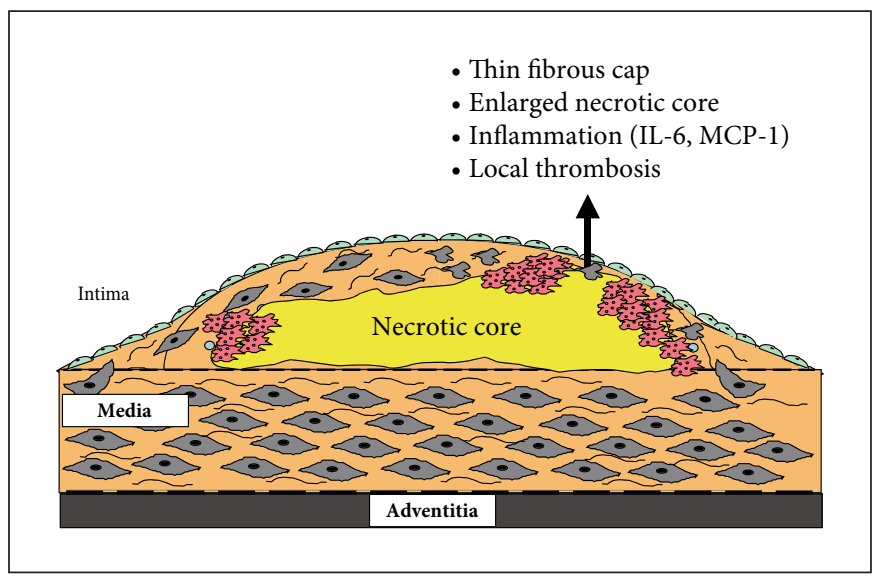

Fig. 1. Direct consequences of VSMC apoptosis. Apoptosis of VSMCs in the fibrous cap (shown as grey cells in the intima) causes thinning of the fibrous cap and expansion of the underlying necrotic core. Apoptotic VSMCs also cause local release of MCP-1 and IL-6, resulting in both recruitment and activation of macrophages (shown in red) to the site of apoptosis. The local inflammation may further weaken the fibrous cap, by secretion of matrix metalloproteinases for instance. The thinned cap is then prone to rupture. Apoptotic VSMCs can also promote both local thrombosis and a systemic procoagulant state, for example by release of microparticles.

are to be predicted, and do not imply that either process is unimportant in disease progression. Small changes in cell division or death could have huge changes in plaque biology bearing this time scale in mind. This study demonstrates that even low levels of detectable apoptosis can induce profound changes in features of vulnerability in atherosclerosis. VSMC apoptosis, particularly if associated with defective clearance, induces inflammation, but even the accumulation of inflammatory cells cannot clear the debris. This emphasises that VSMC apoptosis is an important target to develop therapy to both prevent and treat unstable atherosclerosis.

The study also opens up the possibility of examining the effects of VSMC apoptosis over longer periods of time, and in different stages of the disease. Although apoptotic frequencies peak in advanced plaques, low-level VSMC apoptosis may also profoundly affect plaque development. The conventional view would suggest that reducing VSMCs that contribute to the plaque would reduce plaque formation, akin to reducing restenosis by anti-proliferative therapy. In contrast, plaque growth in humans is characterised by recurrent rounds of silent plaque rupture and repair [23]. If apoptosis triggers these events, particularly if associated with inflammation, 
VSMC apoptosis may exacerbate rather than reduce plaque development. The precise role of VSMCs at different stages of atherogenesis is important, as patients exhibit plaques at varied stages of disease. Therapies aimed at preventing apoptosis in advanced lesions would be unattractive if they accelerated lesion formation in earlier stages of disease.

Although apoptosis alone did not induce aneurysm formation in either normal vessels or plaques, human arterial aneurysm formation occurs mostly in association with atherosclerosis, and medial VSMC loss is associated with profound local inflammation [12]. This mouse model allows the contribution of apoptosis to be assessed in the presence of other stimuli, such as inflammation and hypertension. Similarly, there is extensive in vitro evidence that human VSMC apoptosis can promote calcification in vessels, which is seen both in the intima in advanced plaques and in the media in chronic renal failure. Apoptotic VSMCs may act as both a nidus for calcification, and actively concentrate both calcium and phosphate to generate hydroxyapatite $[16,24]$. This model allows the separate components of VSMC apoptosis and mineral concentrations to be manipulated to dissect the mechanism of arterial calcification in vivo.

As we move to drugs that affect plaque behaviour rather than plaque size, these mice may also provide a useful screen for preclinical testing of drugs that are designed to prevent plaque rupture or help stabilise already vulnerable lesions. To date, there has been no small animal model of plaque rupture that recapitulates all the features of human unstable or ruptured plaques. Most models either involve surgical manipulation or cumbersome delivery of genetic material [25], and in many cases, features of plaque vulnerability take many months to appear [2629]. Whilst this model does not demonstrate lumen thrombosis or myocardial infarction, in part because of the anatomical localisation of plaques in ApoE knockout mice and their efficient fibrinolytic system, multiple features of plaque vulnerability can be induced within 4 weeks of starting DT therapy, without any other intervention. We consider these mice to be a more robust mechanism to examine plaque stabilisation than models that rely on studying atherogenesis or, worse, models that rely on inhibiting neointima formation in normal arteries.

The model also provides a useful tool for determining the role of VSMC apoptosis in other vascular pathologies, such as remodelling $[10,11,30]$, restenosis $[31,32]$ and angiogenesis. In all of these pathologies, VSMC apoptosis has been demonstrated to occur, in some cases with high frequencies. However, the direct role of apoptosis is un- known as the models are frequently associated with other manipulations, such as changes in blood flow or vessel injury. The highly selective and conditional induction of VSMC apoptosis means that the temporal sequence of consequences can be studied, without the use of agents that induce apoptosis in all cell types. In this way, we can determine the direct role of VSMC apoptosis in vascular disease.

\section{Conclusions}

In humans, atherosclerosis is a disease that takes 3040 years to manifest. Until recently, it was not clear what the significance of the relatively low frequencies of VSMC apoptosis was in human atherosclerosis. In addition, although VSMC apoptosis has been implicated in a variety of human vascular diseases, its precise role, in the absence of other stimuli, was unknown. Our recent study has helped clarify the clinical significance of VSMC apoptosis in normal vessels and atherosclerotic plaques. In particular, VSMC apoptosis recapitulates multiple features of plaque vulnerability, identifying manipulation of apoptosis to be an important target in plaque stabilisation.

\section{Acknowledgement}

M.C. and M.B. are supported by BHF grants PG/06/024/20354 and $\mathrm{CH} / 2000003$. 


\section{References}

1 Sata M, Saiura A, Kunisato A, Tojo A, Okada S, Tokuhisa T, Hirai H, Makuuchi M, Hirata Y, Nagai R: Hematopoietic stem cells differentiate into vascular cells that participate in the pathogenesis of atherosclerosis. Nat Med 2002;8:403-409.

-2 Caplice NM, Bunch TJ, Stalboerger PG, Wang S, Simper D, Miller DV, Russell SJ, Litzow MR, Edwards WD: Smooth muscle cells in human coronary atherosclerosis can originate from cells administered at marrow transplantation. Proc Natl Acad Sci USA 2003;100:4754-4759.

-3 Davies M, Richardson P, Woolf N, Katz D, Mann J: Risk of thrombosis in human atherosclerotic plaques: role of extracellular lipid, macrophages, and smooth muscle cell content. Heart 1993;69:377-381.

4 Virmani R, Kolodgie FD, Burke AP, Farb A, Schwartz SM: Lessons from sudden coronary death: a comprehensive morphological classification scheme for atherosclerotic lesions. Arterioscler Thromb Vasc Biol 2000; 20:1262-1275.

5 Ross R: The pathogenesis of atherosclerosis: a perspective for the 1990s. Nature 1993;362: 801-809.

66 Weissberg P, Clesham G, Bennett M: Is vascular smooth muscle cell proliferation beneficial? Lancet 1996;347:305-307.

$\checkmark 7$ Lutgens E, de Muinck ED, Kitslaar PJ, Tordoir JH, Wellens HJ, Daemen MJ: Biphasic pattern of cell turnover characterizes the progression from fatty streaks to ruptured human atherosclerotic plaques. Cardiovasc Res 1999;41:473-479.

8 Geng Y, Libby P: Evidence for apoptosis in advanced human atheroma: colocalization with interleukin-1 $\beta$ converting enzyme. Am J Pathol 1995;147:251-266.

$\checkmark 9$ Littlewood TD, Bennett MR: Apoptotic cell death in atherosclerosis. Curr Opin Lipidol 2003; 14:469-475.

$\checkmark 10$ Cho A, Courtman D, Langille L: Apoptosis (programmed cell death) in arteries of the neonatal lamb. Circ Res 1995;76:168-175.

-11 Cho A, Mitchell L, Koopmans D, Langille BL: Effects of changes in blood flow rate on cell death and cell proliferation in carotid arteries of immature rabbits. Circ Res 1997;81: 328-337.

-12 Lopez-Candales A, Holmes DR, Liao SX, Scott MJ, Wickline SA, Thompson RW: Decreased vascular smooth muscle cell density in medial degeneration of human abdominal aortic aneurysms. Am J Pathol 1997;150: 993-1007.
13 Schaub FJ, Liles WC, Ferri N, Sayson K, Seifert RA, Bowen-Pope DF: Fas and Fas-associated death domain protein regulate monocyte chemoattractant protein-1 expression by human smooth muscle cells through caspase- and calpain-dependent release of interleukin-1 $\alpha$. Circ Res 2003;93:515-522.

14 Schaub FJ, Han DK, Conrad Liles W, Adams LD, Coats SA, Ramachandran RK, Seifert RA, Schwartz SM, Bowen-Pope DF: Fas/ FADD-mediated activation of a specific program of inflammatory gene expression in vascular smooth muscle cells. Nat Med 2000; 6:790-796.

15 Flynn P, Byrne C, Baglin T, Weissberg P, Bennett M: Thrombin generation by apoptotic vascular smooth muscle cells. Blood 1997;89:4373-4384.

16 Proudfoot D, Skepper JN, Hegyi L, Bennett MR, Shanahan CM, Weissberg PL: Apoptosis regulates human vascular calcification in vitro: evidence for initiation of vascular calcification by apoptotic bodies. Circ Res 2000 ; 87:1055-1062.

17 Mallat Z, Hugel B, Ohan J, Leseche G, Freyssinet JM, Tedgui A: Shed membrane microparticles with procoagulant potential in human atherosclerotic plaques - A role for apoptosis in plaque thrombogenicity. Circulation 1999;99:348-353.

18 Mallat Z, Benamer H, Hugel B, Benessiano P, Steg P, Freyssinet J, Tedgui A: Elevated levels of shed membrane microparticles with procoagulant potential in the peripheral circulating blood of patients with acute coronary syndromes. Circulation 2000;101:841-843.

19 Clarke MC, Figg N, Maguire JJ, Davenport AP, Goddard M, Littlewood TD, Bennett MR: Apoptosis of vascular smooth muscle cells induces features of plaque vulnerability in atherosclerosis. Nat Med 2006;12:10751080 .

20 Bennett M, Gibson D, Schwartz S, Tait J: Binding and phagocytosis of apoptotic rat vascular smooth muscle cells is mediated in part by exposure to phosphatidylserine. Circ Res 1995;77:1136-1142.

21 Schrijvers DM, De Meyer GR, Kockx MM, Herman AG, Martinet W: Phagocytosis of apoptotic cells by macrophages is impaired in atherosclerosis. Arterioscler Thromb Vasc Biol 2005;25:1256-1261.

22 Hutter R, Valdiviezo C, Sauter BV, Savontaus M, Chereshnev I, Carrick FE, Bauriedel G, Luderitz B, Fallon JT, Fuster V, Badimon JJ: Caspase- 3 and tissue factor expression in lipid-rich plaque macrophages: evidence for apoptosis as link between inflammation and atherothrombosis. Circulation 2004;109: 2001-2008.
23 Burke AP, Kolodgie FD, Farb A, Weber DK, Malcom GT, Smialek J, Virmani R: Healed plaque ruptures and sudden coronary death: evidence that subclinical rupture has a role in plaque progression. Circulation 2001;103: 934-940.

24 Proudfoot D, Davies JD, Skepper JN, Weissberg PL, Shanahan CM: Acetylated low-density lipoprotein stimulates human vascular smooth muscle cell calcification by promoting osteoblastic differentiation and inhibiting phagocytosis. Circulation 2002;106: 3044-3050.

25 von der Thusen JH, van Vlijmen BJ, Hoeben RC, Kockx MM, Havekes LM, van Berkel TJ, Biessen EA: Induction of atherosclerotic plaque rupture in apolipoprotein E-/- mice after adenovirus-mediated transfer of p53. Circulation 2002;105:2064-2070.

26 Calara F, Silvestre M, Casanada F, Yuan N, Napoli C, Palinski W: Spontaneous plaque rupture and secondary thrombosis in apolipoprotein E-deficient and LDL receptor-deficient mice. J Pathol 2001;195: 257-263.

27 Johnson JL, Jackson CL: Atherosclerotic plaque rupture in the apolipoprotein $\mathrm{E}$ knockout mouse. Atherosclerosis 2001;154: 399-406.

-28 Johnson J, Carson K, Williams H, Karanam S, Newby A, Angelini G, George S, Jackson $\mathrm{C}$ : Plaque rupture after short periods of fat feeding in the apolipoprotein E-knockout mouse: model characterization and effects of pravastatin treatment. Circulation 2005;111: 1422-1430.

29 Rosenfeld ME, Polinsky P, Virmani R, Kauser K, Rubanyi G, Schwartz SM: Advanced atherosclerotic lesions in the innominate artery of the ApoE knockout mouse. Arterioscler Thromb Vasc Biol 2000;20:25872592.

30 Cho A, Langille BL: Arterial smooth-muscle cell turnover during the postnatal period in lambs. FASEB J 1993;7:A756.

31 Isner J, Kearney M, Bortman S, Passeri J: Apoptosis in human atherosclerosis and restenosis. Circulation 1995;91:2703-2711.

32 Bauriedel G, Schluckebier S, Hutter R, Welsch U, Kandoff R, Lüderitz B, Prescott M: Apoptosis in restenosis versus stable-angina atherosclerosis. Arterioscler Thromb Vasc Biol 1998;18:1132-1139. 